\title{
CARACTERÍSTICAS QUíMICAS DO SOLO E ESTRUTURA DE UM FRAGMENTO DE FLORESTA ESTACIONAL SEMIDECIDUAL EM IPIAÇU, Minas Gerais, BRASIL
}

\author{
André Eduardo Gusson ${ }^{1,2}$, Sérgio de Faria Lopes ${ }^{3}$, Olavo Custódio Dias Neto ${ }^{3}$, \\ Vagner Santiago do Vale ${ }^{3}$, Ana Paula de Oliveira ${ }^{3} \&$ Ivan Schiavini ${ }^{3}$
}

\begin{abstract}
Resumo
(Características químicas do solo e estrutura de um fragmento de floresta estacional semidecidual em Ipiaçu, Minas Gerais, Brasil) As florestas estacionais semideciduais comportam grande biodiversidade e encontramse altamente ameaçadas pelo processo de fragmentação. O presente trabalho descreve a estrutura fitossociológica das espécies arbóreas, assim como as classifica pelas síndromes de dispersão e grupos sucessionais, em um fragmento de floresta estacional semidecidual no município de Ipiaçu, MG. Para tanto foram alocadas 25 parcelas de $20 \times 20 \mathrm{~m}$, a partir das quais foram amostrados 837 indivíduos, distribuídos em 25 famílias e 50 espécies, sendo Fabaceae a família mais representativa, com 15 espécies. Hymenaea courbaril foi a espécie com maior VI (18\%), seguida por Piptadenia gonoacantha (10,7\%) e Luehea grandiflora $(10,6 \%)$. Das espécies listadas, $58 \%$ são pioneiras, $21 \%$ secundárias iniciais e $21 \%$ secundárias tardias; $58 \%$ zoocóricas, $33 \%$ anemocóricas e $17 \%$ autocóricas. No fragmento constatou-se que o solo é ácido, com alto teor de $\mathrm{Fe}$ e $\mathrm{Mn}$, com saturação por bases abaixo de 50\%. A baixa riqueza de espécies (50 spp.) e o valor reduzido de área basal $\left(15,15 \mathrm{~m}^{2} \cdot \mathrm{ha}^{-1}\right)$, aliados à alta densidade de espécies pioneiras e/ou oportunistas e às inúmeras clareiras formadas devido a ações antrópicas ao longo do tempo, principalmente o corte seletivo para extração de madeira, são forte indicadores de perturbação na área de estudo.
\end{abstract}

Palavras-chave: fitossociologia, remanescente de floresta, síndrome de dispersão, Fazenda Tucumã.

\section{Abstract}

(Soil chemistry and structure in a fragment of seasonal semidecidous forest in Ipiaçu, Minas Gerais, Brazil) Seasonal semideciduous forests present high biodiversity and are currently threatened by the process of fragmentation. This paper describes the phytosociological structure of woody species in a fragment of seasonal semideciduous forest in the municipality of Ipiaçu, MG, and classify the species by their dispersal syndromes and successional groups. Twenty five plots of 20x20 were allocated, and 837 individuals were sampled representing 50 species distributed in 25 families. Fabaceae was the most representative family with 15 species. Hymenaea courbaril was the species with highest VI (18\%), followed by Piptadenia gonoacantha $(10.7 \%)$ and Luehea grandiflora $(10.6 \%)$. Of the listed species 58\% are pioneers, $21 \%$ first secondary and $21 \%$ late secondary; of these, $58 \%$ are zoochoric, $33 \%$ anemochoric and $17 \%$ autochoric. The fragment has an acid soil with a high content of $\mathrm{Fe}$ and $\mathrm{Mn}$, base saturation below 50\%. The low species richness (50 spp.) and small basal area $\left(15,15 \mathrm{~m}^{2} \cdot \mathrm{ha}^{-1}\right)$, the high density of pioneers and opportunistic species and the presence of several gaps formed by human activity over time, mainly selective cutting of valuable timber trees are all strong signs of the disturbance within the study area.

Key words: dispersal syndromes, forest remnant, phytosociology, Tucumã farm.

\section{INTRODUÇÃo}

Os fragmentos florestais abrigam diversidade biológica ainda desconhecida e altamente ameaçada (Lovejoy \& Bierregaard 1990). Nas últimas décadas houve avanço considerável nos estudos sobre comunidades florestais, devido à sua importância quanto à conservação da diversidade biológica (OliveiraFilho et al. 1994a).

Minas Gerais é considerado um dos estados com maior variedade de formações vegetais do país, devido à ocorrência de três importantes biomas e suas zonas de contato - Cerrado, Mata Atlântica e Caatinga (Drummond et al. 2005).

Artigo recebido em 07/2008. Aceito para publicação em 02/2009.

${ }^{1}$ Instituto de Biologia, Universidade Federal de Uberlândia, R. Ceará s/nº, bloco 2D, s/57, Campus Umuarama, 38400-902, Uberlândia, MG, Brasil.

${ }^{2}$ Autor para correspondência: desrp4@yahoo.com.br

${ }^{3}$ Programa de Pós-graduação em Ecologia e Conservação de Recursos Naturais, Universidade Federal de Uberlândia, Campus Umuarama, bloco 2D, 38400-902, Uberlândia, MG, Brasil. 
O intenso processo de alterações ocorrido ao longo do tempo, o processo histórico de ocupação do território e a conseqüente descaracterização das formações vegetais, influenciado pelas atividades agropecuárias, extrativismo vegetal e produção mineral, reduziram drasticamente a cobertura vegetal natural a fragmentos esparsos. Atualmente, pouco mais de 7,6 milhões de hectares de cobertura florestal estão distribuídos entre as bacias do Rio Grande e Rio Paranaíba, com destaque para os fragmentos de florestas estacionais semideciduais, com 1.305.777 ha (Scolforo \& Carvalho 2006).

Esses fragmentos, por serem mais susceptíveis à ação antrópica, ao efeito de borda e a desastres naturais, geralmente apresentam alterações na estrutura e dinâmica da sua comunidade arbórea, de maneira que merecem atenção especial (Willians-Linera 1990; Laurance \& Yensen 1991). Assim, estudos detalhados sobre a riqueza das espécies e a ecologia das comunidades vegetais são fundamentais para embasar quaisquer iniciativas de preservação e conservação dos fragmentos florestais (Oliveira-Filho et al. 1994a).

Especificamente na região do Triângulo Mineiro, a expansão da fronteira agrícola, sobretudo da agricultura e pecuária para exportação, reduziu as florestas estacionais semideciduais a pequenos fragmentos. Por esta razão, o objetivo do presente estudo foi caracterizar o solo, descrever e analisar a riqueza das espécies e a estrutura horizontal e vertical da comunidade arbórea em um fragmento florestal situado no Município de Ipiaçu, no Pontal do Triângulo Mineiro.

\section{Materiais e Métodos \\ Caracterização da área de estudo}

O estudo foi conduzido em um fragmento de floresta localizado na Fazenda Tucumã, município de Ipiaçu, região do Pontal do Triângulo, Minas Gerais, cujas coordenadas centrais são $18^{\circ} 43^{\prime} \mathrm{S}$ e 4956'W. O fragmento tem área total de cerca de 40 ha, localizada a uma altitude de aproximadamente $530 \mathrm{~m}$ a.n.m. Segundo o sistema proposto por Veloso et al. (1991), o fragmento classifica-se como floresta estacional semidecidual. O clima da região é classificado com Aw, pelo sistema de Köppen, apresentando duas estações bem definidas, um período chuvoso e um período seco, com temperatura média anual de $23,3^{\circ} \mathrm{C}$ e pluviosidade média anual de $1528 \mathrm{~mm}$. Durante a estação seca, que se estende de junho a setembro, a temperatura e pluviosidade médias são reduzidas para $20,2^{\circ} \mathrm{C}$ e $23 \mathrm{~mm}$, respectivamente (Estação Climatológica de Capinópolis, MG - fonte INMET).

A área está inserida em um mosaico de pastagens, culturas anuais e outros fragmentos florestais, maiores e menores que o fragmento em estudo, e apresenta-se impactada devido à alterações ocorridas ao longo do tempo, causadas principalmente pelo corte seletivo para retirada de madeira. O cultivo da cana-deaçúcar foi implantado no entorno do fragmento no início do ano de 2006. A comunidade vegetal é formada, em sua maioria, por indivíduos jovens de espécies arbóreas e/ou espécies de pequeno porte, com a presença de poucos indivíduos de grande porte. O fragmento apresenta grande número de clareiras naturais e antrópicas, em sua maioria ocasionadas pelo corte seletivo de madeira na área.

\section{Amostragem fitossociológica e caracterização do solo}

O presente estudo foi realizado durante o período de setembro de 2006 a janeiro de 2007. Para o estudo fitossociológico em florestas tropicais, recomenda-se a utilização de no mínimo um hectare de área amostral, segundo o protocolo da Rede de Parcelas Permanentes dos Biomas Cerrado e Pantanal (Felfili et al. 2005). Foram alocadas 25 parcelas, com distribuição sistemática e contígua de $20 \times 20$ metros. Em cada parcela, foram registrados e identificados todos os indivíduos arbóreos vivos que apresentaram circunferência à altura do peito (CAP $) \geq 15$ $\mathrm{cm}$. As medidas de altura foram estimadas por 
comparação com a altura de uma tesoura de poda alta de $12 \mathrm{~m}$. Indivíduos que apresentavam troncos múltiplos tiveram as CAPs medidas individualmente. Assim, foi calculado um diâmetro único para estes indivíduos a partir dos valores de circunferências, para fins de distribuição entre classes de tamanho. $\mathrm{O}$ material botânico coletado foi identificado por especialistas ou comparado com coleções de herbário. O material testemunho para todas as espécies foi depositado no Herbarium Uberlandensis (HUFU), da Universidade Federal de Uberlândia, MG. Foi utilizado o sistema de classificação do Angiosperm Phylogeny Group II (APG II). As espécies foram distribuídas em três grupos sucessionais: pioneiras, secundárias iniciais e secundárias tardias, tomando-se por base os trabalhos realizados por Gandolfi et al. (1995), Paula et al. (2004) e Catharino et al. (2006), além de observações no campo.

As espécies foram classificadas quanto à síndrome de dispersão, adotando-se os critérios morfológicos dos frutos, definidos por Pijl (1982) e com auxílio da literatura (Pinheiro \& Ribeiro 2001). Dessa forma, os diásporos foram classificados em três grupos: anemocóricos (dispersão por vento), zoocóricos (dispersão por animais) e autocóricos (dispersão por gravidade e/ou explosiva).

Os parâmetros fitossociológicos de densidade, dominância e frequência relativas e o valor de importância (VI) foram analisados, utilizando-se o programa Fitopac Shell, versão 1.6.4 (Shepherd 2006). A avaliação da diversidade alfa foi obtida por meio dos índices de diversidade de Shannon (H') e de equabilidade de Pielou (J').

Para o estudo da estrutura horizontal da comunidade utilizou-se a distribuição de frequiência das classes em diâmetro fixas em $5 \mathrm{~cm}$ de amplitude. Foi realizada a distribuição de frequência dos indivíduos nas classes diamétricas para a comunidade como um todo, bem como para as espécies com maior VI de cada grupo sucessional (pioneira, secundária inicial e secundária tardia). Para a distribuição dos indivíduos na estrutura vertical aplicou-se a fórmula proposta por Spiegel (1977), estabelecendo-se o número e intervalo das classes de altura, com a primeira classe incluindo todos os indivíduos com altura até $4,07 \mathrm{~m}$.

Para a caracterização do solo foram feitas apenas análises de propriedades químicas. Foram coletadas dez amostras compostas, a partir de três amostras simples, retiradas na profundidade de 0 a $20 \mathrm{~cm}$ nos limites (inferior direito, centro e superior esquerdo) das parcelas selecionadas para coleta $(1,3,5,7,9,11,13,15,17$ e 23). As amostras foram submetidas a análises químicas de acordo com a rotina utilizada no Laboratório de Solos (LAS) da Universidade Federal de Uberlândia. Utilizando-se o Sistema Brasileiro de Classificação de Solos (Embrapa 2006) e por meio dos resultados das análises químicas propôs-se uma classificação para o solo amostrado.

\section{Resultados e Discussão Análise do solo}

Os resultados das análises químicas com seus parâmetros estão apresentados na Tabela 1. $\mathrm{O}$ solo amostrado apresenta $\mathrm{pH}$ 5,0. Para a Embrapa (2006), solos que possuam $\mathrm{pH}$ inferior a 6/6,5 são considerados ácidos. Segundo Raij (1991), solos com essas características são denominados ácidos devido ao baixo teor de cátions, como $\mathrm{Ca}, \mathrm{Mg}, \mathrm{K}$ e $\mathrm{Na}$.

A região de Cerrado abrange grande parte do território mineiro, ocorrendo mosaicos de formações florestais com destaque para as florestas estacionais semideciduais (Rizzini 1997), onde sua distribuição coincide com solos mais férteis do que as formações savânicas do entorno, úmidos e profundos, muito visados pela agricultura para cultivo de monoculturas (Oliveira-Filho et al. 1994b). No entanto, a saturação por bases $(\mathrm{V})$ da análise permaneceu abaixo de 50\%, com altos teores de Fe e Mn, indicando um solo com baixa disponibilidade de macronutrientes e classificado, para fins de comparação, como distrófico, segundo o critério estabelecido pela Embrapa (2006). Entretanto, 
Tabela 1 - Composição química de solo $(0-20 \mathrm{~cm}) \mathrm{em}$ fragmento de floresta estacional semidecidual no município de Ipiaçu, Minas Gerais. $\mathrm{P}=$ fósforo; $\mathrm{K}^{+}=$potássio; $\mathrm{Ca}^{2+}=$ cálcio; $\mathrm{Mg}^{2+}=$ magnésio; $\mathrm{Al}^{3+}=$ alumínio; $\mathrm{SB}=$ soma de bases; $\mathrm{T}=$ capacidade de troca catiônica em $\mathrm{pH} 7 ; \mathrm{Fe}=$ ferro; $\mathrm{Mn}$ = manganês; $\mathrm{V}=$ saturação por bases; $\mathrm{MO}=$ matéria orgânica; d.p. = desvio padrão; $\mathrm{CV}=$ coeficiente de variação.

Table 1 - Soil chemical and nutrient composition $(0-20 \mathrm{~cm})$ from seasonal semidecidual forest remnants at the municipality of Ipiaçu, Minas Gerais. $\mathrm{P}=$ phosphorous; $\mathrm{K}^{+}=$potassium; $\mathrm{Ca}^{2+}=$ calcium; $\mathrm{Mg}^{2+}=$ magnesium; $\mathrm{Al}^{3+}=$ aluminum $\mathrm{SB}=$ sum of bases; $\mathrm{T}=$ cation exchange capacity at $\mathrm{pH} 7 ; \mathrm{Fe}=$ iron; $\mathrm{Mn}=$ manganese; $\mathrm{V}=$ base saturation; $\mathrm{MO}=$ organic matter; d.p. = standard deviations; $\mathrm{CV}=$ coeficient of variation.

\begin{tabular}{|c|c|c|c|c|c|c|c|c|c|c|c|c|}
\hline & \multirow[b]{3}{*}{ pH } & \multicolumn{7}{|c|}{ Cátions trocáveis } & \multicolumn{4}{|c|}{ Micronutrientes } \\
\hline & & \multicolumn{2}{|c|}{$\mathrm{mg} \mathrm{dm}^{-3}$} & \multicolumn{5}{|c|}{ Cmolc dm ${ }^{-3}$} & \multicolumn{2}{|c|}{$\mathbf{m g d m}^{-3}$} & & \multirow{2}{*}{$\begin{array}{c}\begin{array}{c}\text { dag } \\
\mathbf{k g}^{-1}\end{array} \\
\text { MO }\end{array}$} \\
\hline & & $\mathbf{P}$ & $\mathbf{K}^{+}$ & $\mathbf{C a}^{2+}$ & $\mathbf{M g}^{2+}$ & $\mathbf{A l}^{3+}$ & SB & $\mathbf{T}$ & $\mathrm{Fe}$ & Mn & & \\
\hline Média & 5,02 & 7,23 & 35,3 & 1,12 & 0,44 & 0,18 & 1,66 & 4,18 & 34,40 & 28,60 & 38,80 & 1,62 \\
\hline Mínimo & 4,80 & 3,30 & 25,00 & 0,40 & 0,20 & 0,00 & 0,70 & 3,50 & 15,00 & 22,00 & 19,00 & 1,20 \\
\hline Máximo & 5,50 & 31,30 & 52,00 & 2,20 & 0,60 & 0,30 & 2,90 & 5,10 & 59,00 & 36,00 & 57,00 & 1,90 \\
\hline Amplitude & 0,70 & 28,00 & 27,00 & 1,80 & 0,40 & 0,30 & 2,20 & 1,60 & 44,00 & 14,00 & 38,00 & 0,70 \\
\hline d.p. & 0,25 & 8,49 & 7,17 & 0,51 & 0,13 & 0,10 & 0,62 & 0,46 & 11,95 & 4,33 & 11,29 & 0,204 \\
\hline CV & 0,05 & 1,17 & 0,20 & 0,45 & 0,29 & 0,57 & 0,37 & 0,11 & 0,347 & 0,15 & 0,291 & 0,126 \\
\hline
\end{tabular}

mesmo em áreas próximas, as florestas estacionais semideciduais ocorrem sobre solos com diferentes concentrações de nutrientes, maiores e menores que a encontrada para o fragmento estudado em Ipiaçu, como verificado no trabalho desenvolvido por Kilca (2007), em uma área no município de Uberlândia, e reportado por Vale (2008), para um remanescente estudado no município de Araguari, ambos também no Triângulo Mineiro.

\section{Fitossociologia}

Foram identificadas 26 famílias, 43 gêneros e 50 espécies arbóreas, em 837 indivíduos amostrados (Tab. 2). A densidade absoluta foi de 837 indivíduos por hectare, área basal de $15,15 \mathrm{~m}^{2} / \mathrm{ha}$ e altura média de $6,9 \mathrm{~m}$. Estes valores de riqueza e área basal podem ser indicativos de alterações na área, uma vez que são considerados baixos, quando comparados com a média de outros estudos em florestas estacionais semideciduais, que é de 117 espécies e $29 \mathrm{~m}^{2} /$ ha (Vale 2008). O baixo valor encontrado para área basal em Ipiaçu pode estar relacionado a algum tipo de perturbação ocorrido no passado, como o corte seletivo de indivíduos arbóreos de grande porte, fato registrado durante o levantamento de dados.

As famílias que mais contribuíram para a riqueza de espécies foram: Fabaceae, com 13 espécies (26\%); Rubiaceae, com quatro espécies (8\%); Myrtaceae com três espécies (6\%); e Anacardiaceae, Apocynaceae, Boraginaceae, Euphorbiaceae, Malvaceae, Meliaceae, Sapindaceae e Cannabaceae com duas espécies cada (32\%). O restante das famílias (28\%) apresentou apenas uma espécie.

O índice de Shannon (H') calculado foi de 2,94 e a equabilidade ( $\left.\mathrm{J}^{\prime}\right)$ de 0,74 , valores considerados baixos para florestas estacionais semideciduais, quando comparados com outros estudos nessas formações, realizados com o mesmo esforço amostral. Vale (2008), analisando 15 fragmentos de florestas estacionais semideciduais no sudeste do Brasil, obteve média de $3,59( \pm 0,5)$ e $0,78( \pm 0,04)$ respectivamente para os índices de Shannon e equalibidade. Alguns trabalhos realizados em 
Tabela 2 - Espécies amostradas e seus parâmetros fitossociológicos em fragmento de floresta estacional semidecidual, Ipiaçu, MG. DR = densidade relativa; DoR = dominância relativa; FR = frequência relativa; $\mathrm{GS}$ = Grupo sucessional $(\mathrm{P}=$ pioneira; $\mathrm{SI}=$ secundária inicial; $\mathrm{ST}$ = secundária tardia e Scla = sem classificação); SD = Síndrome de dispersão (Ane=anemocórica; Auto=autocórica; Zoo=zoocórica); VI = valor de importância.

Table 2 - Plant species surveyed, with its respective phytosociological parameters, on seasonal semidecidual forest remnants at the municipality of Ipiaçu, Minas Gerais $\mathrm{DR}=$ relative density; $\mathrm{DoR}=$ relative dominance; $\mathrm{FR}=$ relative frequency; GS = sucessional group; $(\mathrm{P}=$ pioneer; $\mathrm{SI}=$ initial secondary; $\mathrm{ST}=$ late secondary; $\mathrm{Scla}=$ without classification); $\mathrm{SD}=$ dispersion syndrome (Ane=anemocory; Auto=autocory; $\mathrm{Zoo}=\mathrm{zoocory}$ ); $\mathrm{VI}=$ importance value.

\begin{tabular}{|c|c|c|c|c|c|c|c|c|}
\hline Espécie & Família & SD & GS & NI & DR & DoR & $\mathbf{F R}$ & IVI \\
\hline Hymenaea courbaril L. & Fabaceae & Zoo & ST & 63 & 7,53 & 41,91 & 6,27 & 55,70 \\
\hline $\begin{array}{l}\text { Piptadenia gonoacantha (Mart.) } \\
\text { J.F. Macbr }\end{array}$ & Fabaceae & Auto & $P$ & 159 & 19,00 & 6,56 & 6,84 & 32,40 \\
\hline Luehea grandiflora Mart. \& Zucc. & Malvaceae & Ane & $\mathrm{P}$ & 133 & 15,89 & 9,68 & 6,55 & 32,13 \\
\hline Casearia gossypiosperma Briq. & Salicaceae & Ane & SI & 65 & 7,77 & 3,22 & 6,84 & 17,82 \\
\hline Guapira areolata (Heimerl) Lundell & Nyctaginaceae & Zoo & SI & 44 & 5,26 & 2,96 & 4,84 & 13,06 \\
\hline Copaifera langsdorffii Desf. & Fabaceae & Zoo & ST & 19 & 2,27 & 6,20 & 4,56 & 13,03 \\
\hline $\begin{array}{l}\text { Protium heptaphyllum (Aubl.) } \\
\text { Marchand }\end{array}$ & Burseraceae & Zoo & SI & 20 & 2,39 & 5,93 & 3,42 & 11,74 \\
\hline Alibertia sessilis (Vell.) K. Schum. & Rubiaceae & Zoo & ST & 41 & 4,90 & 1,38 & 4,84 & 11,12 \\
\hline Trichillia catigua A. Juss. & Meliaceae & Zoo & ST & 40 & 4,78 & 1,38 & 4,56 & 10,72 \\
\hline $\begin{array}{l}\text { Campomanesia velutina (Cambess) } \\
\text { O. Berg. }\end{array}$ & Myrtaceae & Zoo & SI & 31 & 3,70 & 1,64 & 5,13 & 10,48 \\
\hline Machaerium brasiliensis Vogel & Fabaceae & Ane & SI & 32 & 3,82 & 1,61 & 4,56 & 10,00 \\
\hline Celtis iguanae (Jacq.) Sarg. & Cannabaceae & Zoo & $\mathrm{P}$ & 22 & 2,63 & 1,72 & 4,56 & 8,91 \\
\hline Apuleia leiocarpa (Vogel) J.F. Macbr. & Fabaceae & Ane & SI & 18 & 2,15 & 2,72 & 3,13 & 8,00 \\
\hline $\begin{array}{l}\text { Agonandra brasiliensis Miers } \\
\text { ex Benth. \& Hook. f. }\end{array}$ & Opiliaceae & Zoo & ST & 17 & 2,03 & 2,07 & 2,85 & 6,95 \\
\hline Sweetia fruticosa Spreng. & Fabaceae & Ane & ST & 15 & 1,79 & 0,87 & 3,42 & 6,08 \\
\hline $\begin{array}{l}\text { Albizia niopoides (Spruce ex Benth.) } \\
\text { Burkart }\end{array}$ & Fabaceae & Ane & $\mathrm{P}$ & 18 & 2,15 & 1,26 & 2,56 & 5,97 \\
\hline Myracrodruon urundeuva Allemão & Anacardiaceae & Ane & ST & 11 & 1,31 & 1,19 & 2,56 & 5,07 \\
\hline $\begin{array}{l}\text { Astronium fraxinifolium Schott } \\
\text { ex Spreng }\end{array}$ & Anacardiaceae & Ane & ST & 8 & 0,96 & 0,35 & 1,99 & 3,30 \\
\hline Margaritaria nobillis L. f. & Phyllanthaceae & Auto & SI & 8 & 0,96 & 0,25 & 1,71 & 2,91 \\
\hline Psidium sartorianum (O. Berg) Nied. & Myrtaceae & Zoo & SI & 6 & 0,72 & 0,29 & 1,71 & 2,71 \\
\hline $\begin{array}{l}\text { Terminalia glabrescens (Cambess. } \\
\text { exA. St.-Hil.) Eichler }\end{array}$ & Combretaceae & Ane & ST & 5 & 0,60 & 0,39 & 1,42 & 2,41 \\
\hline Pouteria gardneri Ducke & Sapotaceae & Zoo & ST & 5 & 0,60 & 0,09 & 1,42 & 2,11 \\
\hline Sapium glandulatum (Vell) Pax. & Euphorbiaceae & Zoo & $\mathrm{P}$ & 3 & 0,36 & 0,81 & 0,85 & 2,02 \\
\hline Dipteryx alata Vogel & Fabaceae & Zoo & SI & 2 & 0,24 & 1,15 & 0,57 & 1,96 \\
\hline Bauhinia rufa (Bong.) Steud. & Fabaceae & Auto & SI & 5 & 0,60 & 0,21 & 1,14 & 1,95 \\
\hline Ceiba speciosa A. St.-Hil. & Malvaceae & Ane & SI & 2 & 0,24 & 1,05 & 0,57 & 1,86 \\
\hline $\begin{array}{l}\text { Enterolobium contortisiliquum } \\
\text { (Vell) Hauman }\end{array}$ & Fabaceae & Auto & $\mathrm{P}$ & 2 & 0,24 & 0,91 & 0,57 & 1,72 \\
\hline Bauhinia ungulata L. & Fabaceae & Auto & $\mathrm{P}$ & 5 & 0,60 & 0,19 & 0,85 & 1,64 \\
\hline Coutarea hexandra (Jacq.) K. Schum. & Rubiaceae & Ane & SI & 5 & 0,60 & 0,17 & 0,85 & 1,62 \\
\hline
\end{tabular}




\begin{tabular}{lllllllll}
\hline Espécie & Família & SD & GS & NI & DR & DoR & FR & IVI \\
\hline Matayba guianensis Aubl. & Sapindaceae & Zoo & SI & 3 & 0,36 & 0,22 & 0,85 & 1,43 \\
$\begin{array}{l}\text { Aspidosperma cylindrocarpon } \\
\text { M. Arg. }\end{array}$ & Apocynaceae & Ane & SI & 3 & 0,36 & 0,18 & 0,85 & 1,40 \\
Roupala brasiliensis Klotzsch & Proteaceae & Ane & ST & 4 & 0,48 & 0,33 & 0,57 & 1,38 \\
Magonia pubescens A. St.-Hil. & Sapindaceae & Auto & SI & 2 & 0,24 & 0,17 & 0,57 & 0,98 \\
Trema micrantha (L.) Blume & Cannabaceae & Zoo & P & 2 & 0,24 & 0,06 & 0,57 & 0,87 \\
Guettarda virbunoides Cham. & Rubiaceae & Zoo & SI & 2 & 0,24 & 0,05 & 0,57 & 0,86 \\
\& Schltdl. & & & & & & & & \\
Ixora brevifolia Benth. & Rubiaceae & Zoo & ST & 2 & 0,24 & 0,05 & 0,57 & 0,86 \\
Cordia sp1 L. & Boraginaceae & Zoo & Scla & 2 & 0,24 & 0,05 & 0,57 & 0,85 \\
Ocotea sp1 Aubl. & Lauraceae & Zoo & Scla & 1 & 0,12 & 0,32 & 0,28 & 0,72 \\
Coccoloba mollis Casar. & Polygonaceae & Zoo & SI & 1 & 0,12 & 0,10 & 0,28 & 0,51 \\
Platypodium elegans Vogel & Fabaceae & Ane & SI & 1 & 0,12 & 0,08 & 0,28 & 0,49 \\
Trichillia elegans A. Juss. & Meliaceae & Zoo & ST & 1 & 0,12 & 0,04 & 0,28 & 0,44 \\
Rollinia sylvatica (A.St.-Hil.) Mart. & Annonaceae & Zoo & SI & 1 & 0,12 & 0,03 & 0,28 & 0,43 \\
Aspidosperma subincanum Mart. & Apocynaceae & Ane & ST & 1 & 0,12 & 0,03 & 0,28 & 0,43 \\
Rhamnidium elaeocarpum Reissek & Rhamnaceae & Zoo & P & 1 & 0,12 & 0,02 & 0,28 & 0,43 \\
Não identificada 1 & Scla & Scla & Scla & 1 & 0,12 & 0,02 & 0,28 & 0,43 \\
Não identificada 2 & Scla & Scla & Scla & 1 & 0,12 & 0,03 & 0,28 & 0,43 \\
Boraginaceae 1 & Boraginaceae & Scla & Scla & 1 & 0,12 & 0,02 & 0,28 & 0,42 \\
Acacia polyphylla DC. & Fabaceae & Auto & P & 1 & 0,12 & 0,02 & 0,28 & 0,42 \\
Myrcia splendens (Sw.) DC. & Myrtaceae & Zoo & P & 1 & 0,12 & 0,01 & 0,28 & 0,42 \\
Aloysia virgata (Ruiz \& Pav.) Juss. & Verbenaceae & Ane & SI & 1 & 0,12 & 0,02 & 0,28 & 0,42 \\
\hline
\end{tabular}

Minas Gerais apresentaram índices de diversidade que variam de 3,62 a 4,28 e equabilidade de 0,78 a 0,91 (Souza et al. 2003; Silva et al. 2003; Silva et al. 2004). Os baixos índices encontrados no presente estudo, sobretudo para H', devem-se ao fato do fragmento apresentar-se em estádio inicial de sucessão, com regeneração pós-perturbação. Neste caso, o esperado é que a comunidade apresente baixa riqueza de espécies e que algumas poucas espécies possuam alta densidade. Segundo Viana et al. (1992), o tamanho, a forma, o grau de isolamento, o tipo de vizinhança e o histórico de perturbações se manifestam e se combinam, gerando diferentes formas de degradação, umas das quais é a perda da diversidade.

Hymenaea courbaril, a espécie com maior VI $(18,6 \%)$, exibiu também a maior dominância relativa (42\%) devido à elevada circunferência à altura do peito (CAP), superior a $300 \mathrm{~cm}$ em alguns indivíduos. O mesmo foi observado para Copaifera langsdorffii e Protium heptaphyllum. As demais espécies com alto VI destacaram-se principalmente por apresentarem alta densidade, como Piptadenia gonoacantha, Luehea grandiflora, Casearia gossypiosperma, Guapira areolata, Alibertia sessilis, Trichilia catigua, Campomanesia velutina e Machaerium brasiliense.

Apesar da ocorrência predominante de espécies típicas da florestas estacionais semideciduais, o fragmento apresenta algumas espécies também encontradas em outras fitofisionomias. Platypodium elegans (1), Dipterix alata (2), Magonia pubescens (2), Guetarda viburnoides (2), Astronium fraxinifolium (8) e Myracrodruon urundeuva (11) ocorrem freqüentemente em cerradão (Costa \& Araujo 2001; Saporetti et al. 2003; Gomes et al. 2004; Salis et al. 2006). A ocorrência dessas espécies em diferentes 
fisionomias se deve ao fato das formações florestais no Cerrado estarem originalmente associadas às formações savânicas, formando regiões de transição savana-floresta nas quais muitas de espécies de uma formação permanecem no interior da outra, sobretudo nesses pontos de contato (Guilherme \& Nakajima 2007).

A alta densidade de Piptadenia gonoacantha pode estar relacionada ao seu caráter de espécie pioneira, apresentando-se tolerante a diferentes níveis de luminosidade (Ferreira et al. 2001). O fragmento estudado apresenta inúmeras clareiras espalhadas pelas parcelas, a maioria causada por alterações antrópicas no ambiente, facilitando assim o estabelecimento de espécies pioneiras. Oliveira-Filho et al. (1998) consideram a luminosidade que penetra pelas clareiras como o principal fator relacionado às alterações na distribuição e abundância de espécies lenhosas em florestas decíduas do Brasil Central.

A distribuição dos indivíduos em 14

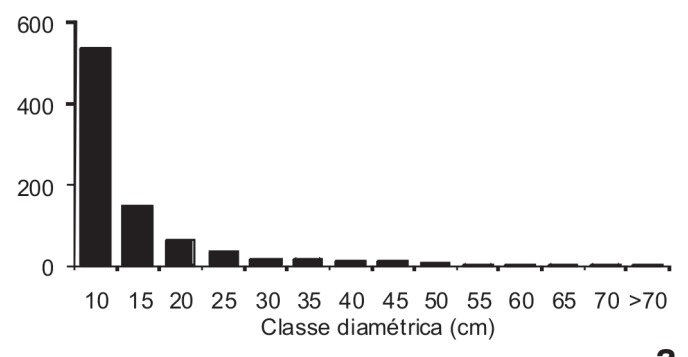

a

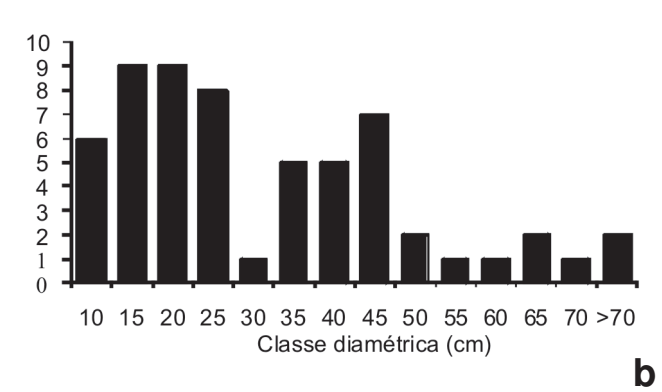

b

classes de diâmetro foi feita para a comunidade como um todo, assim como para as espécies Hymenaea courbaril (secundária tardia), Casearia gossypiosperma (secundária inicial) e Piptadenia gonoacantha (pioneira), representando as de maior VI em cada grupo sucessional (Fig. 1a-d). A distribuição diamétrica para a comunidade revelou que a maioria dos indivíduos (89\%) está incluso nas três menores classes de DAP (Fig. 1a). Esse padrão "J reverso" demonstra que a comunidade arbórea apresenta muitos indivíduos jovens ou de pequeno porte nas primeiras classes e/ou no componente de regeneração natural, com poucos indivíduos de poucas espécies compondo o dossel do fragmento. A maior concentração de indivíduos nas primeiras classes de diâmetro pode caracterizar uma comunidade estoque, com idade e composição de espécies variadas (Scolforo et al. 1998).

Hymenaea courbaril (secundária tardia) apresenta uma distribuição equitativa
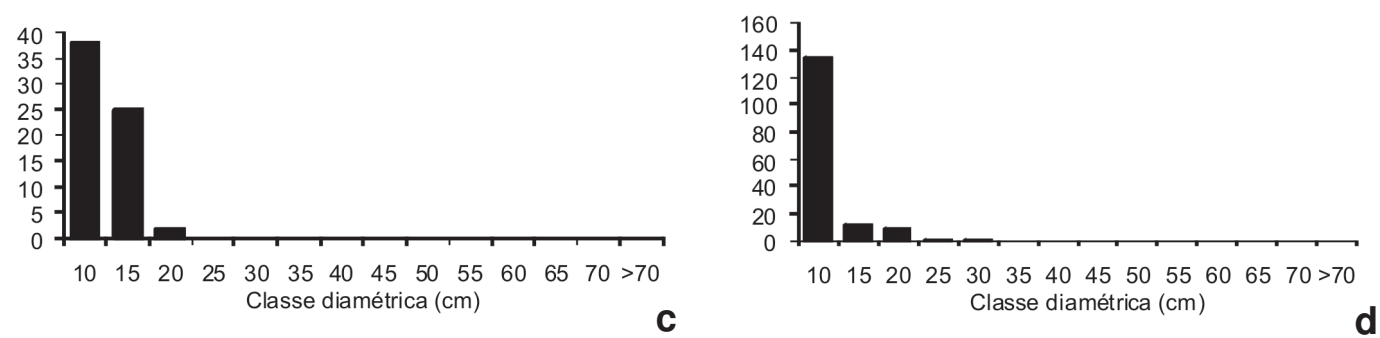

Figura 1 -Distribuição dos indivíduos em classes de diâmetro, em fragmento de floresta estacional semidecidual em Ipiaçu, Minas Gerais. a - total de indivíduos amostrados; b - Hymenaea courbaril; c - Casearia gossypiosperma; d-Piptadenia gonoacantha.

Figure 1 - Individuals distributed according to its respective diameter class, on seasonal semidecidual forest remnants at the municipality of Ipiaçu, Minas Gerias. a - total sum of the individuals surveyed; b - Hymenaea courbaril; c - Casearia gossypiosperma; d - Piptadenia gonoacantha. 
entre as classes de diâmetro, com indivíduos em todas as classes. No entanto, apresenta poucos indivíduos que alcançam diâmetro superior a $50 \mathrm{~cm}$ e, mesmo não apresentando padrão "J" reverso, mostra-se estável na comunidade (Fig. 1b). Casearia gossypiosperma (secundária inicial) e Piptadenia gonoacantha (pioneira) demonstram maior concentração dos indivíduos na primeira classe de diâmetro (Fig. 1c-d). Entretanto, a primeira concentra também grande parte de seus indivíduos na segunda classe diamétrica.

Os diferentes padrões na distribuição das espécies corroboram as características ecológicas do grupo sucessional em que se classificam, principalmente $P$. gonoacantha, com alta capacidade de colonizar ambientes perturbados. Apesar de Gandolfi et al. (1995) considerarem que espécies pioneiras são claramente dependentes de condições de maior luminosidade nas fases iniciais de desenvolvimento, não ocorrendo, em geral, no subosque, para Carvalho (1994) P. gonoacantha tolera condições de sombreamento parcial, podendo assim permanecer no subosque até a abertura de clareiras, quando, então, crescem rapidamente. Esta situação parece ser corroborada pelo comportamento de $P$. gonoacantha observado em campo, devido ao grande número de indivíduos jovens presentes no subosque da área estudada.

O processo de perturbação antrópica ocorrido no fragmento, associado às características ecológicas de espécies pioneiras, facilitou que estas espécies obtivessem sucesso no seu estabelecimento. Espécies pioneiras possuem facilidade em estabelecerem-se em sítios alterados, melhoram a fertilidade do solo e facilitam o estabelecimento de outras espécies, geralmente são de crescimento rápido, com ciclo de vida curto, e restritas às clareiras (Gandolfi et al. 1995). No caso do fragmento em estudo, a maioria das clareiras foi gerada pelo corte seletivo de madeira de espécies de grande porte, como $H$. courbaril e $C$. langsdorffii, segundo o relato dos proprietários, o que parece ter favorecido a colonização por espécies pioneiras.
A distribuição dos indivíduos da comunidade em classes de altura demonstra um subosque denso, com altura média de $6,9 \mathrm{~m} \mathrm{e}$ poucos indivíduos de grande porte compondo o dossel do fragmento (Fig. 2). O subosque apresenta domínio das espécies Piptadenia gonoacantha, Luehea grandiflora, Casearia gossypiosperma, Campomanesia velutina e Alibertia sessilis, enquanto no dossel do fragmento predominam Hymenaea courbaril, Copaifera langsdorffii, Protium heptaphyllum e Apulea leiocarpa.

Das 50 espécies listadas, $48 \%$ são zoocóricas (39\% dos indivíduos), 32\% anemocóricas (39\% dos indivíduos), 14\% autocóricas (22\% dos indivíduos) e 6\% sem classificação (Fig. 3 a,c). Para as florestas tropicais, espera-se uma percentagem maior em zoocoria, com uma dominância entre $70 \%$ e $90 \%$ estimada para as espécies, de acordo com a literatura científica. A baixa ocorrência de espécies zoocóricas apresentada no fragmento Ipiaçu pode ser um indicativo de pouca atividade da fauna dispersora, fato que pode comprometer o recrutamento de novos indivíduos nas populações, um processo essencial para a manutenção das espécies. Em relação aos grupos sucessionais, $40 \%$ das espécies foram classificadas como secundárias iniciais (30\% dos indivíduos), 28\% secundárias tardias ( $28 \%$ dos indivíduos), $22 \%$ pioneiras (41\% dos indivíduos) e 10\% sem classificação (1\% dos indivíduos) (Fig. 3 b,d). Henriques

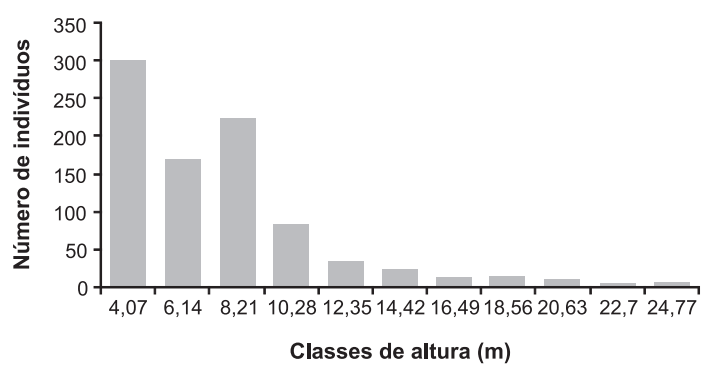

Figura 2 - Distribuição dos indivíduos em classes de altura, em comunidade arbórea amostrada em fragmento de floresta estacional semidecidual em Ipiaçu, Minas Gerais.

Figure 2 - Individuals distributed according to its respective height class, on the tree community of seasonal semidecidual forest remnants at the municipality of Ipiaçu, Minas Gerais. 

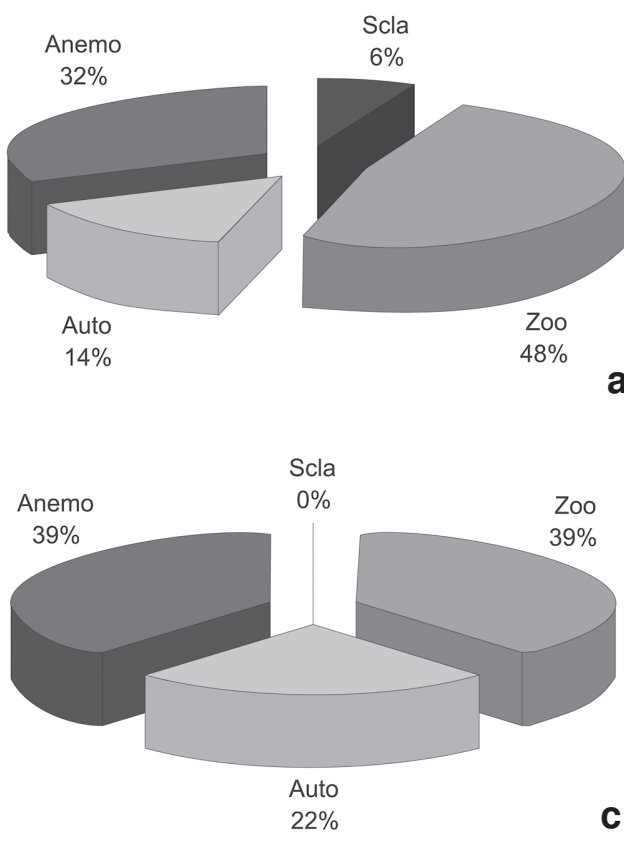
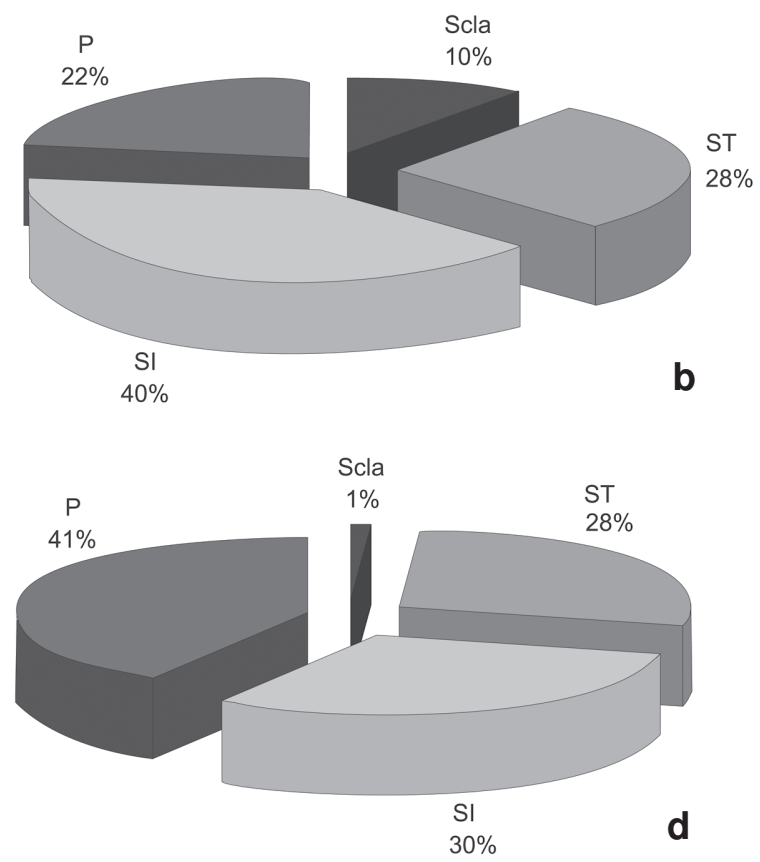

Figura 3 - Distribuição das espécies e indivíduos amostrados na comunidade arbórea em fragmento de Floresta Estacional Semidecidual em Ipiaçu, MG. a - Proporção das espécies pela síndrome de dispersão; b - Proporção de espécies por classe sucessional; c - Proporção de indivíduos pela síndrome de dispersão; d - Proporção de indivíduos por classe sucessional. $\mathrm{P}=$ Pioneira, $\mathrm{SI}=$ Secundária inicial, $\mathrm{ST}=$ Secundária tardia ; Anemo = Anemocoria, Auto = Autocoria, Zoo = Zoocoria, Scla $=$ Sem classificação.

Figure 3 - Distribution of plant species and individuals on seasonal semidecidual forest remnants at the municipality of Ipiaçu, Minas Gerias a - Proportion of species according to its respective dispersion syndrome; b - Proportion of species according to its respective sucessional class; $\mathrm{c}$ - Proportion of individuals according to its respective dispersion syndrome; $\mathrm{d}$ - Proportion of individuals according to its respective sucessional class. $\mathrm{P}=$ Pioneer, $\mathrm{SI}=$ initial secondary, $\mathrm{ST}=$ late secondary Anemo $=$ Anemocory, Auto $=$ Autocory, Zoo $=$ Zoocory, Scla $=$ without classification.

(2003) considera que, em geral, predominam nos fragmentos as espécies secundárias iniciais e zoocóricas, com uma tendência em aumentar a proporção de espécies pioneiras e anemocóricas, à medida que aumentam as perturbações antrópicas.

Os resultados obtidos neste estudo corroboram alguns dados propostos por Henriques (2003) e contradizem outros, sobretudo quando se considera a abundância das espécies. O fragmento estudado em Ipiaçu não apresenta alta riqueza de espécies anemocóricas e nem de pioneiras, mesmo sendo um fragmento com grande perturbação. No entanto, quando analisada a densidade relativa dessas espécies, percebe-se que os indivíduos das espécies pioneiras dominam o estrato arbóreo na comunidade ( $41 \%$ do total, Fig. 3d), sugerindo que a perturbação de um fragmento não é indicada apenas pelo número de espécies pioneiras e/ou anemocóricas presentes, devendo-se levar em consideração também a abundância dessas espécies nos fragmentos.

A partir dos resultados apresentados, pode-se dizer que o fragmento de floresta estudado apresenta-se fortemente alterado, onde as espécies pioneiras e secundárias iniciais representam $62 \%$ da riqueza e $71 \%$ da densidade total, sendo $40 \%$ das espécies e $30 \%$ dos indivíduos classificados como secundários iniciais. Segundo Gandolfi et al. (1995), a porcentagem elevada de espécies secundárias iniciais é explicada pelo histórico de fragmentação, perturbação e deciduidade periódica de uma certa porcentagem de espécies, sendo que o predomínio dessas indica mau estado de conservação. 
A predominância de Piptadenia gonoacantha, que nas florestas do Triângulo Mineiro pode ser considerada uma espécie oportunista em ambientes perturbados, confirma o baixo nível de conservação do fragmento. O grande número de clareiras, a maioria formada pelo corte seletivo de madeira, favoreceu o estabelecimento e a predominância de indivíduos de espécies pioneiras, principalmente Piptadenia gonoacantha e Luehea grandiflora. A baixa eqüabilidade mostra também distribuição heterogênea das espécies no fragmento, demonstrando baixa riqueza de espécies e alta densidade de indivíduos de espécies pioneiras. Uma análise realizada a partir apenas da riqueza de espécies pioneiras e/ou anemocórias é insuficiente para se determinar o grau de perturbação de um fragmento, devendo-se levar em consideração a densidade das populações dessas espécies.

\section{REFERÊNCIAS BIBLIOGRÁFICAS}

APG II. 2003. An update of the Angiosperm Phylogeny Group classification for the orders and families of flowering plants: APG II. Botanical Journal of the Linnean Society 41: 399-436.

Carvalho, P. E. R. 1994. Espécies florestais brasileiras: recomendações silviculturais, potencialidades e uso da madeira. EMBRAPA - CNPF, Brasília, 640p.

Catharino, E. L. M.; Bernacci, L. C.; Franco, G. A. D. C.; Durigan, G. \& Metzger, J. P. 2006. Aspectos da composição e diversidade do componente arbóreo das florestas da Reserva Florestal do Morro Grande, Cotia, SP. Biota Neotropica 6(2) Disponível em: http:// www.biotaneotropica.org.br/v6n2/pt/ abstract?article+bn00306022006. Acessado em: 25/06/2007

Costa, A. A. \& Araújo, G. M. 2001. Comparação da vegetação arbórea de Cerradões e de Cerrado na Reserva do Panga, Uberlândia, Minas Gerias. Acta Botanica Brasilica 15 (1): 63-72.
Drummond, G. M.; Martins, C. S.; Machado, A. B. M.; Sebaio, F. A. \& Antonini, Y. 2005. Biodiversidade em Minas Gerais: um atlas para sua conservação. Fundação Biodiversitas. Belo Horizonte. $2^{a}$ ed. 222 p.

Embrapa. Centro Nacional de Pesquisa de Solos. 2006. Sistema brasileiro de classificação de solos (SiBCS). Embrapa Solos. Rio de Janeiro. $2^{\mathrm{a}}$ ed., 316p.

Felfili, M. F.; Carvalho, F. A. \& Haidar, R. F. 2005. Manual para monitoramento de parcelas permanentes nos biomas Cerrado e Pantanal. Brasília: Universidade de Brasília, 55p.

Ferreira, J. N.; Ribeiro, J. F. \& Lazarini da Fonseca, C. E. 2001. Crescimento inicial de Piptadenia gonoacantha (Leguminosae, Mimosoideae) sob inundação em diferentes níveis de luminosidade. Revista Brasileira de Botânica 24(4): 561-566.

Gandolfi, S.; Leitão Filho, H. F. \& Bezerra, C. L. F. 1995. Levantamento florístico e caráter sucessional das espécies arbustivoarbóreas de uma floresta semidecídua no município de Guarulhos, SP. Revista Brasileira de Biologia 55: 753-767.

Gomes, B. Z.; Martins, F. R. \& Tamashiro, J. Y. 2004. Estrutura do cerradão e da transição entre cerradões e floresta paludícola num fragmento da International Paper do Brasil Ltda., em Brotas, SP. Revista Brasileira de Botânica 27(2): 249-262.

Guilherme F. A. G. \& Nakajima, J. N. 2007. Estrutura da vegetação arbórea de um remanescente ecotonal urbano florestasavana no Parque do Sabiá, em Uberlândia, MG. Revista Árvore 31(2): 329-337.

Henriques, O. K. 2003. Caracterização da vegetação natural de Ribeirão Preto, SP: Bases para conservação. Tese de Doutorado, Universidade de São Paulo, São Paulo, 221p.

Kilca, R. V. 2007. Padrões florísticos, estruturais e relações edáficas entre dois tipos de florestas tropicais estacionais no 
Cerrado. Dissertação de Mestrado, Universidade Federal de Uberlândia, Minas Gerais, 64p.

Laurance, W. F. \& Yensen, E. 1991. Predicting the impacts of edges in fragmented habitats. Biological Conservation 55(1): 77-92.

Lovejoy, T. E. \& Bierregaard, R. O. 1990. Central Amazonian forest and the minimum critical size of ecossystems project. In: Gentry, A. H. (ed.). Four neotropical rainforest. Yale University Press, New Haven. Pp. 60-71.

Oliveira-Filho, A. T.; Almeida, R. J.; Mello, J. M. \& Gavilanes, M. L. 1994a. Estrutura fitossociológica e variáveis ambientais em um trecho de mata ciliar do córrego dos Vilas Boas, Reserva Biológica do Poço Bonito, Lavras (MG). Revista Brasileira de Botânica 17: 67-85.

Oliveira-Filho, A. T.; Vilela E. A.; Carvalho, D. A. \& Gavilanes, M. L. 1994b. Differentiation of streamside and upland vegetation in area of montane semidecidous forest in southeartern Brazil. Flora 189(4): 287-305.

Oliveira-Filho, A. T.; Curi N.; Vilela, E. A. \& Carvalho, D. A. 1998. Effects of canopy gaps, topography, and soils on the distribution of woody species in a Central Brazilian deciduous dry forest. Biotropica 30(3): 362-375.

Paula, A.; Silva, A. F.; Marco-Júnior, P.; Santos, F. A. M. \& Souza, A. L. 2004. Sucessão ecológica da vegetação arbórea em uma floresta estacional semidecidual, Viçosa, MG, Brasil. Acta Botanica Brasilica 18(3): 407-423.

Pinheiro, F. \& Ribeiro, J. F. 2001. Síndromes de dispersão de sementes em mata de galeria no Distrito Federal. In: Ribeiro, J. F.; Fonseca, C. E. L.; Sousa-Silva, J. C. Cerrado: caracterização e recuperação de mata de galeria. EMBRAPA Cerrados, Planaltina. Pp.335-375.

Pijl, L. van der. 1982. Principles of dispersal in higher plants. Springer-Verlag, Berlin, 219p.
Raij, B. V. 1991. Fertilidade do solo e adubação: acidez e calagem. Associação Brasileira para Pesquisa da Potassa e do Fosfato, Piracibaca, 343p.

Rizzini, C. T. 1997. Tratado de fitogeografia do Brasil. Rio de Janeiro. Âmbito Cultural, 330p.

Salis, S. M.; Assis, M. A.; Crispim, S. M. A. \& Casagrande, J. C. 2006. Distribuição e abundância de espécies arbóreas no Pantanal, Mato Grosso do Sul, Brasil. Revista Brasileira de Botânica 29(3): 339-352.

Saporetti Jr, A. W.; Meira Neto, J. A. A. \& Paula Amado, R. 2003. Fitossociologia de cerrado sensu stricto no município de Abaeté, MG. Revista Árvore 27(3): 413-419.

Scolforo, J. R. S.; Pulz, F. A. \& Mello, J. M. 1998. Modelagem da produção, idade das florestas nativas, distribuição espacial das espécies e a análise estrutura. In: Scolforo, J. R. S. Manejo florestal ( org.). UFLA/FAEPE, Lavras. Pp. 189-246.

Scolforo, J. R. \& Carvalho, L. M. T. 2006. Mapeamento e inventário da flora nativa e dos reflorestamentos de Minas Gerais. Universidade Federal de Lavras UFLA, Instituto Estadual de Florestas IEF, 288p.

Shepherd, G. J. 2006. Fitopac Shell 1.6.4. Manual versão preliminar. Campinas. Departamento de Botânica IB da UNICAMP, SP.

Silva, A. F.; Oliveira, R. V.; Santos, N. R. L. \& Paula, A. 2003. Composição florística e grupos ecológicos das espécies de um trecho de floresta semidecídua submontana da Fazenda São Geraldo, Viçosa-MG. Revista Árvore 27(3): 311-319.

Silva, N. R. S.; Martins, S. V. J.; Meira Neto, A. A.; Souza, A. L. 2004. Composição florística e estrutura de uma floresta estacional semidecidual montana em Viçosa, MG. Revista Árvore 28(3): 397-405.

Souza, J. S.; Espirito-Santo, F. D. B.; Fontes, M. A. L.; Oliveira-Filho, A. T. \& Bortezelli, L. 2003. Análise das variações florísticas e estruturais da comunidade arbórea de um fragmento de floresta semidecídua às 
margens do rio Capivari, Lavras-MG. Revista Árvore 27(2): 185-206.

Spiegel, M. R. 1977. Estatística. São Paulo. McGraw-Hill do Brasil. 580p.

Vale, V. S. 2008. Padrões e processos ecológicos do componente arbóreo em uma área de floresta estacional semidecidual (Araguari, MG). Dissertação de Mestrado. Universidade Federal de Uberlândia, Minas Gerais, 91p.

Veloso, H. P.; Rangel Filho, A. L. R. \& Lima, J. C. A. 1991. Classificação da vegetação brasileira adaptada a um sistema universal. FIBGE, Rio de Janeiro, 123p.

Viana, V. M.; Tabanez. A. A. J. \& Martins, J. L. A. 1992. A restauração e manejo de fragmentos florestais. In: Congresso Nacional sobre Essências Nativas, vol. 2, São Paulo, 1992. Anais. Instituto Florestal de São Paulo, São Paulo. Pp. 400-407.

Willians-Linera, G. 1990. Vegetation structure and environmental conditions of forest edges in Panama. Journal of Ecology 78(2): 356-373. 